Markus Nussbaumer

\title{
Notrecht in der Coronakrise
}

Kurzbericht von der 19. wissenschaftlichen Tagung des Zentrums für Rechtsetzungslehre, Universität Zürich, 9. September 2020

Beitragsart: Tagungsberichte

Zitiervorschlag: Markus Nussbaumer, Notrecht in der Coronakrise, in: LeGes 31 (2020) 3 
[1] Die Leitung des Zürcher Zentrums für Rechtsetzungslehre (ZfR) hat schnell reagiert und schon im Frühling 2020 das Thema der für den September vorgesehenen alljährlichen wissenschaftlichen Tagung kurzerhand ausgewechselt und durch die alles dominierende Thematik der «Notrechtsetzung» ersetzt. Und sie hat Mut, Standfestigkeit und Innovationsgeist bewiesen, war doch sehr lange unklar, ob und wie eine solche Tagung durchgeführt werden könnte. Die anfängliche Hoffnung, bis im September 2020 würden sich Vorbilder für solche Tagungen finden, von denen man abschauen und lernen könnte, hat sich zerschlagen: Die ZfR-Tagung wurde tatsächlich zur Pionierin - niemand hatte es bis dahin gewagt, eine solche Veranstaltung in diesem Ausmass durchzuführen (und heute, Anfang November 2020, könnte sie nicht mehr durchgeführt werden). Es wurde eine hybride Veranstaltung, mit etwa 100 Teilnehmerinnen und Teilnehmern insgesamt, wovon circa 30 Personen online zugeschaltet wurden, während die andern physisch vor Ort an der Universität Zürich waren. Der technische Aufwand, die nicht physisch anwesenden Personen in die Plenarveranstaltungen und dann in die einzelnen Workshops zuzuschalten, war immens, und der Verlauf trotz aller Schwierigkeiten erstaunlich gut, unkompliziert, hoch professionell und für alle Beteiligten fruchtbar.

[2] Schnell reagiert hat übrigens nicht bloss die ZfR-Leitung, sondern ein guter Teil der akademischen juristischen Zunft, waren doch schon im Vorfeld der Tagung und in erstaunlich kurzer Zeit eine ganze Reihe wissenschaftlicher Beiträge zum aktuellen Notrecht (nebst sehr vielen Stellungnahmen in den Medien) erschienen, die auch für die ZfR-Tagung gleichsam die Hintergrundfolie oder den Teppich bildeten. ${ }^{1}$ Es scheint, als hätte der Lockdown den akademisch tätigen Juristinnen und Juristen eine willkommene Verschnaufpause von den Verpflichtungen in Lehre und akademischer Selbstverwaltung verschafft, eine Verschnaufpause, die einige sehr produktiv für Reflexionen darüber nutzten, was da in der Rechtsetzung gerade «so abging».

[3] Das Setting der Tagung war das gleiche wie in früheren Jahren: Plenarvorträge und Workshops. Drei Plenarvorträge gab es am Vormittag: Bernhard Waldmann (Uni FR) eröffnete mit den staatsrechtlichen Grundlagen und Herausforderung von «Notrecht» (es stellte sich übrigens schnell heraus, dass dieser Begriff in der aktuellen Krise unterschiedlich und oft zu weit verwendet wird). Felix Uhlmann (Uni ZH und ZfR) folgte mit den verwaltungsrechtlichen Aspekten und Herausforderungen, und schliesslich STEFAN HöFler mit den legistischen Eigenheiten und Herausforderungen der aktuellen Covid-19-Verordnungsgebung des Bundesrates. Wer diesen Vorträgen in der Aula der Uni ZH folgte und es sich nicht ganz verkneifen konnte, gelegentlich auf dem Handy die letzten News zu lesen (nicht weil die Vorträge langweilig waren, aber weil viele der Teilnehmerinnen und Teilnehmer quasi aus brennenden Häusern davongeeilt und nach Zürich gefahren waren), konnte etwas erleben, was es so noch kaum je gab: Während vorne die Referenten über Gegenstände vortrugen wie das im Entstehen begriffene Covid-19-Gesetz, kamen über den Newsticker laufend Abstimmungsresultate zu ebendiesem Gesetz (das die Bundesversammlung schliesslich am 25. September 2020 verabschiedet und auf den nächstfolgenden Tag in Kraft gesetzt hat). Wann hat man je erlebt, dass Wissenschaft zeitlich so nah am rechts-

1 Erwähnt seien nebst anderen: Giovanni Biaggins: «Notrecht» in Zeiten des Coronavirus - Eine Kritik der jüngsten Praxis des Bundesrats zu Art. 185 Abs. 3 BV, ZBl 121 (2020), 5, S. 239 ff.; die Ausgabe 6/2020 der AJP unter anderem mit dem Beitrag von Florian Brunner/Martin Wilhelm/Felix Uhlmann: Das Coronavirus und die Grenzen des Notrechts. Überlegungen zu einer ausserordentlichen ausserordentlichen Lage, S. 685 ff., und mit dem Beitrag von Stefan Höfler: Notrecht als Krisenkommunikation? Redaktionelle Fallgruben in der COVID19-Verordnung 2, S. 702 ff.; die Sondernummer 2020 der ZSR zum Thema Pandemie und Recht; der Sammelband COVID-19. Ein Panorama der Rechtsfragen zur Corona-Krise. Basel: Helbing\&Lichtenhahn; das Interview mit Markus Müller (Uni BE) und Susanne Kuster (stv. Direktorin des BJ) im plädoyer 3/2020, S. 7 ff.; und viele mehr. 
politischen Geschehen ist. Manchmal hätte man am liebsten neben die Powerpoint-Folien des Vortrags in der Aula der Universität Zürich das Live-Streaming aus dem Parlamentsgebäude in Bern projiziert.

[4] Die Rechtsetzungslehre hat unter anderem die Funktion, die Rechtsetzungspraxis von Bundesrat und Parlament kritisch zu beleuchten. Zahlreich waren die kritischen wissenschaftlichen Stimmen zur Coronagesetzgebung im Vorfeld der Tagung. Es war denn auch nicht unbedingt zu erwarten und tat insgesamt doch gut zu hören und zu sehen, dass bei aller Kritik die Arbeit der rechtsetzenden Behörden in diesem Land, vor allem des Bundesrates, der ja in die Coronakrise recht eigentlich «hineingestürchelt» ist und in der ausserordentlichen Lage dann praktisch alles an sich zog, gar nicht so schlecht wegkam. Die Funktion einer solchen Tagung ist jedoch nicht, einfach zu loben, sondern auf die Schwachstellen hinzuweisen und erste Lehren zu ziehen. Und das tat sie auch, und dies wohltuend ruhig, sachlich, kritisch-konstruktiv.

[5] Am Nachmittag gab es vier Workshops, jeder zweimal hintereinander angeboten, sodass die Teilnehmerinnen und Teilnehmer (vor Ort oder online zugeschaltet) zwei der vier Workshops besuchen konnten. Angeboten wurde (A) ein Erfahrungsbericht aus dem Gesundheitsbereich, von Mike Sсhüpbach und Marion Stauffer, Bundesamt für Gesundheit (BAG), also aus dem Bundesamt, das wie kein anderes seit Ausbruch der Krise im Dauerstress und im Schaufenster von Öffentlichkeit und Politik steht; (B) ein Erfahrungsbericht aus dem Bildungsbereich, von FranzisKa Gschwend (Bildungsdepartement des Kantons St. Gallen); (C) ein Erfahrungsbericht aus dem Polizeibereich von August Mächler (Universität ZH); sowie (D) ein Erfahrungsbericht aus der Rechtsetzungsbegleitung der Bundesverwaltung, von Markus NussBaumer (Bundeskanzlei, BK) und Miriam Sahlfeld (Bundesamt für Justiz, BJ), die Rechtsetzungsprojekte des Bundes zum einen materiell-rechtlich prüfen und begleiten (BJ) und zum andern auch sprachlichredaktionell (als Verwaltungsinterne Redaktionskommission, VIRK, bestehend aus BK und BJ).

[6] Die Referate und die Zusammenfassungen der Workshops werden wie jedes Jahr in einem Tagungsband (Erscheinungsdatum voraussichtlich Sommer 2021) nachzulesen sein.

[7] 2021 wird das ZfR seine 20. Jahrestagung durchführen. Zu dieser Jubiläumstagung hat sich das Zentrum die «mehrsprachige Gesetzgebung» als Thema vorgenommen. Stattfinden soll die Konferenz, wie es sich für ein Jubiläum gehört, erstmals an zwei Tagen, am 13. und 14. September 2021. Wir hoffen doch sehr, dass es bei diesem Thema bleibt und sich die Leitung des ZfR nicht gezwungen sieht, wiederum kurzfristig thematisch umzuschwenken auf «Notrecht in der Coronakrise 2.0». Und wir hoffen, es wird eine ganz «gewöhnliche» Tagung mit physischer Präsenz und ohne Masken. Die Hoffnung stirbt ja bekanntlich zuletzt.

Markus Nussbaumer, Bundeskanzlei. 